\title{
The Effect of Flow Circulation on the Scattering of Landing Gear Noise
}

\author{
Timothy Heffernon, David Angland ${ }^{\dagger}$ Xin Zhang ${ }^{\ddagger}$ and Malcolm Smith ${ }^{\S}$ \\ Faculty of Engineering and the Environment, \\ University of Southampton, Southampton, Hampshire, SO16 7QF, UK.
}

\begin{abstract}
An investigation into the scattering of landing gear noise sources by a lifting wing is presented. A two-dimensional test case is used in the investigation. The noise sources are represented by a monopole, which is located at the approximate position of a landing gear underneath a wing geometry. A linearized Euler equation solver is used to simulate the scattering of the monopole by the wing. The effect of a non-uniform flowfield due to circulation induced by a lifting wing is quantified as the difference in acoustic scattering over uniform and non-uniform base flows. The results show that increasing the angle of attack, or increasing the Mach number, leads to a small increase in the sound pressure level towards the ground. However, the observed increase is relatively small. A boundary element method solver is then used to investigate the same problem, and existing uniform and non-uniform flow boundary element formulations are evaluated to see which more accurately predicts the effect of flow. The results show that the uniform flow boundary element formulation is more accurate in predicting the effect of flow than the non-uniform flow approximation for this particular problem, and that the extra computational effort required for the non-uniform flow approximation does not yield a more accurate result.
\end{abstract}

\section{Nomenclature}

Symbols

a airfoil chord length

$b \quad$ boundary angle function

c speed of sound

$F \quad$ monopole forcing region function

$f \quad$ acoustic frequency

$G \quad$ Green's function

$H_{n}^{(1)} \quad$ Hankel function of the first kind of order $n$

$i \quad$ complex unit

$k \quad$ acoustic wavenumber

$M \quad$ Mach number

n normal vector

$n$ normal

$p \quad$ pressure

$Q \quad$ monopole strength parameter

$r \quad$ distance between two points

S monopole source function

$S \quad$ surface

$T$ Taylor-transformed time $t$ time

u velocity vector

$v \quad$ velocity mangitude

X Taylor-transformed position vector

$\mathbf{x}$ position vector

$\mathbf{y}$ position vector

Greek Symbols

$\alpha \quad$ angle of attack

$\beta \quad$ monopole forcing region parameter

$\delta \quad$ delta function

$\epsilon \quad$ monopole amplitude parameter

$\theta \quad$ observer angle

$\kappa \quad$ modified acoustic wavenumber

$\lambda \quad$ acoustic wavelength

$\rho$ density

$\Phi_{a} \quad$ time-dependent acoustic potential

$\phi_{a} \quad$ time-harmonic acoustic potential

$\phi_{f} \quad$ flow potential

${ }^{*}$ PhD student, Airbus Noise Technology Centre, Student Member AIAA.

${ }^{\dagger}$ Lecturer, Airbus Noise Technology Centre, Member AIAA.

¥Airbus Noise Technology Centre. Also Chair Professor, Department of Mechanical and Aerospace Engineering, The Hong Kong University of Science and Technology, Clear Water Bar, Kowloon, Hong Kong SAR, China. Associate Fellow AIAA.

$\S$ Technical Manager, Institute of Sound and Vibration Research Consulting. 
$\omega \quad$ angular frequency

Subscripts

0 mean flow variable

$s \quad$ monopole source

ref reference

rms root mean square

Superscripts

I acoustic variable $\sim \quad$ Lorentz-transformed variable

$\wedge \quad$ Taylor-transformed variable

- Taylor-transformed Helmholtz variable

$I \quad$ incident

$S \quad$ scattered

Abbreviations

BEM boundary element method

LEE linearized Euler equations

SPL sound pressure level

\section{Introduction}

TCREASINGLY stringent noise regulations are being introduced in the vicinity of airports in an effort to Ireduce the environmental impact of the aviation industry. Aircraft manufacturers are required to design their aircraft to ensure that these regulations are met. The European Commission has set a target to reduce perceived noise emissions from aircraft by $65 \%$ by the year 2050 relative to a new aircraft in $2000 .^{1}$

There are two types of noise from aircraft: engine noise and airframe noise. A lot of work has been done since the 1970's on high-bypass turbofan engines and as a result modern engines produce less noise than older designs, to the extent that engine noise on approach is now comparable to airframe noise. ${ }^{2}$ During approach, an aircraft will have landing gears and high-lift devices such as flaps and slats deployed, and these are all major contributors to airframe noise. ${ }^{2}$

The aim of the work presented here was to investigate the scattering of landing gear noise from the underside of a lifting wing with a uniform and non-uniform base flow, and to compare two boundary element method flow formulations that approximate these effects. The investigation was conducted in two dimensions and at a single acoustic frequency as a proof-of-concept. In the future it will be extended to three dimensions, and the effect of frequency on the scattering will be investigated.

A lifting wing induces circulation in a flow, and it was an objective of the investigation to see if and how the circulation affects the scattering of landing gear noise sources. A high-order linearized Euler equation (LEE) solver ${ }^{3}$ was used to compute the scattered sound field around a NACA-0012 airfoil, using a monopole source located at the approximate location of a landing gear. This was computed at various angles of attack and Mach numbers, over both uniform and non-uniform inviscid base flows. This enabled the effect of the non-uniform flowfield due to the circulation induced by the lifting wing to be quantified.

Boundary element method (BEM) solvers for acoustic problems offer certain advantages over domain solvers, primarily a decrease in time required to set up the problem due to the comparative ease of meshing, and the ability to compute the solution in a domain of infinite size. Boundary element methods are more suited to problems in the absence of flow, due to boundary integral formulations relying on the use of Green's functions. However, boundary element formulations that approximate uniform flow ${ }^{4}$ and non-uniform flow ${ }^{5,6}$ effects do exist in the literature. Another aim of the present investigation was to determine which of these approximations is more suitable for simulation of installation effects of landing gears under lifting wings, and boundary element simulations using these two flow approximations were conducted to this end.

\section{Numerical Methods}

A NACA-0012 airfoil was chosen as the scattering body for all numerical simulations. The symmetrical airfoil is non-lifting at zero angle of attack. The airfoil was set as acoustically rigid in all simulations.

Landing gear directivity is almost omnidirectional when small-scale parts that create high frequency noise are removed. ${ }^{2}$ As such, a single monopole source was used to model a simplified landing gear. Given the airfoil chord length $a$, the source was positioned at $(+a / 2,-a / 2)$ from the leading edge. The airfoil geometry and source location can be seen in Figure 1. A chord length of $a=5 \mathrm{~m}$ was chosen to reflect the size of wing found on smaller commercial airliners. A frequency $f=325 \mathrm{~Hz}$ was chosen for this investigation so that the source was representative of a large-scale landing gear component. This choice of chord length and frequency gave a non-dimensional Helmholtz number of $k a=30$, where $k=2 \pi f / c_{0}$ is the acoustic wavenumber with the speed of sound $c_{0}=340.2 \mathrm{~m} / \mathrm{s}$. 
The sound pressure level (SPL) was computed for both LEE and BEM solvers at 360 points on a circle of radius $a$ centered on the source. The $\theta=0^{\circ}$ observer position was located in the negative $x$-direction from the source, with $\theta$ increasing in an anti-clockwise direction. These observer locations can be seen in Figure 1. The SPL was computed using SPL $=20 \log _{10}\left(p_{\text {rms }} / p_{\text {ref }}\right)$, with the reference pressure $p_{\text {ref }}=2.0 \times 10^{-5} \mathrm{~Pa}$.

The circulation around a lifting wing increases as angle of attack $\alpha$ and free stream Mach number $M_{0}$ is increased. To investigate the effect that this has on the acoustic scattering of a monopole, the following simulations were performed:

- A Mach number of $M_{0}=0.2$ was fixed, and $\alpha$ was varied in the range $0^{\circ} \leq \alpha \leq 8^{\circ}$.

- An angle of attack of $\alpha=5^{\circ}$ was fixed, and $M_{0}$ was varied in the range $0.1 \leq M_{0} \leq 0.3$.

In the simulations, the airfoil and source positions remained fixed, and the angle of attack was varied by changing the free stream Mach vector, as shown in Figure 1. This allowed the same mesh to be used for all of the LEE simulations. The range of Mach numbers investigated covered speeds that are representative of a small commercial airliner on approach.

These simulations were conducted with the LEE solver with uniform and non-uniform base flows, and using the BEM solver with both uniform and non-uniform flow approximations. Inviscid non-uniform base flows are required for both the LEE and the non-uniform flow BEM formulation, and this also enables the results from both solvers to be compared. The inviscid flow assumption is approximately valid at all values $0^{\circ} \leq \alpha \leq 8^{\circ}$, as it has been shown that the flow remains attached to the airfoil at these angles of attack. ${ }^{7}$

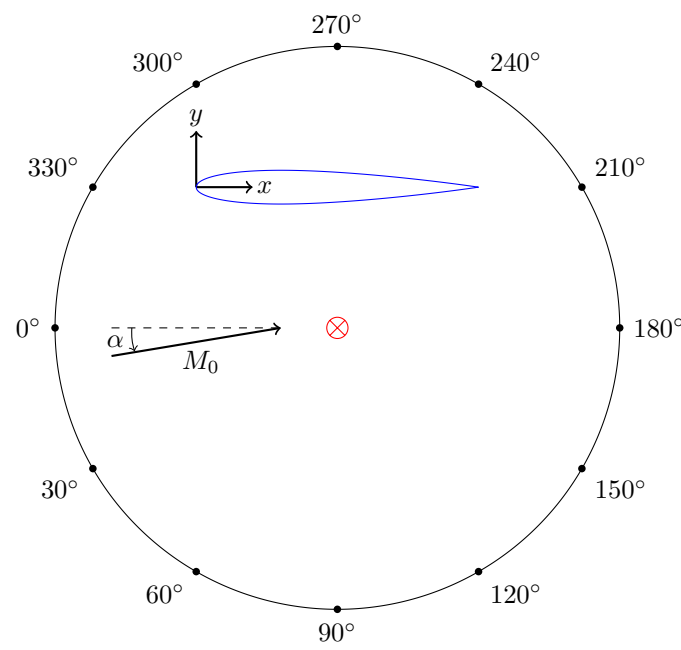

Figure 1. NACA-0012 airfoil geometry, monopole source location, and observer locations.

\section{A. Linearized Euler equation solver}

The linearized Euler equations are given by

$$
\begin{gathered}
\frac{\partial \rho^{\prime}}{\partial t}+\nabla \cdot\left(\rho_{0} \mathbf{u}^{\prime}+\mathbf{u}_{0} \rho^{\prime}\right)=0 \\
\frac{\partial \mathbf{u}^{\prime}}{\partial t}+\left(\mathbf{u}_{0} \cdot \nabla\right) \mathbf{u}^{\prime}+\left(\mathbf{u}^{\prime} \cdot \nabla\right) \mathbf{u}_{0}+\frac{1}{\rho_{0}}\left(\nabla p^{\prime}-\frac{\rho^{\prime}}{\rho_{0}} \nabla p_{0}\right)=0 .
\end{gathered}
$$

Here $p$ is pressure, $\rho$ is density, and $\mathbf{u}$ is a velocity vector. The acoustic variables with superscript $/$ are functions of position vector $\mathbf{x}=(x, y)$, and time $t$. The mean flow variables with subscript 0 are functions of position only, as the flow is assumed to be steady. The solid walls were treated with a slip-wall boundary condition.

A monopole source at a single frequency is implemented in a similar manner to Bailly and Juvé, ${ }^{8}$ by adding a source term to the right hand side of Eq. (1). This term is given by

$$
\mathbf{S}(x, y, t)=\epsilon \omega \sin (\omega t) F(x, y),
$$


with

$$
F(x, y)=\exp \left(-\beta\left[\left(x-x_{s}\right)^{2}+\left(y-y_{s}\right)^{2}\right]\right) .
$$

Here $\omega=2 \pi f$ is the angular frequency with $f$ the frequency of the acoustic wave, $\epsilon$ is the amplitude of the monopole, and $\beta$ controls the width of the monopole forcing region.

The linearized Euler equations are solved using the solver SotonLEE. SotonLEE is a multi-block solver for structured grids that uses a fourth-order compact spatial discretization scheme ${ }^{9,10}$ with a sixth-order compact filtering scheme, ${ }^{11}$ and a fourth-order low dispersion and dissipation Runge-Kutta explicit temporal scheme. ${ }^{12}$ Non-reflecting optimized zonal transverse characteristic boundary conditions are used on the edges of the computational domain to prevent acoustic reflections that would contaminate the acoustic solution. ${ }^{13}$

A structured quadrilateral mesh was created around the NACA-0012 airfoil geometry. The mesh consisted of $1.84 \times 10^{5}$ nodes in 97 blocks. The topology of the mesh is shown in Figure 2. This mesh was extended to a length of $10 a$ in all directions around the airfoil for the base flow solution, to ensure that the pressure far field boundaries were far enough away from the airfoil to allow for an accurate flow solution. The flow mesh consisted of $6.83 \times 10^{5}$ nodes in 140 blocks. The flow mesh in the region surrounding the airfoil was identical to the acoustic mesh shown in Figure 2, so that each required flow solution was known at all nodes of the acoustic mesh for the LEE solver. These two meshes were used for all acoustic and mean flow computations, with the flow angle of attack being specified in the pressure far field boundary conditions in the flow computation. The LEE solver is parallelized, and each acoustic solution was computed in approximately 15 minutes using 16 Intel Xeon cores at $2.6 \mathrm{GHz}$.

To compute the SPL at the observer locations, $p_{\text {rms }}$ was obtained by sampling the time varying pressure at each observer at a rate of 25 samples per period for 10 periods. Initial simulations of a monopole in the free field showed that the radius of the monopole forcing region should be less than $\lambda / 10$, where $\lambda$ is the acoustic wavelength. This ensured that accurate convective amplification effects were observed when the computed SPL was compared to an analytical solution. The average maximum distance between mesh nodes was $0.04 \mathrm{~m}$, which ensured that the acoustic solution was accurately resolved with at least 10 points per wavelength upstream at the highest Mach number under investigation. The mesh was further refined in the source region with a node spacing of $0.02 \mathrm{~m}$ to ensure that the forcing region covered 4 to 5 nodes radially.

Studies showed that the amplitude parameter $\epsilon$ is dependent on several factors, including the mesh and forcing region radius. For each of the simulations, $\epsilon$ was scaled so that the source strength was nominally the same between the LEE and BEM simulations with uniform flow. For the uniform LEE mean flowfield a uniform mean flow was specified everywhere in the domain, except at nodes on the boundary where $x \geq 0.3 a$. The point $x=0.3 a$ is the point of maximum thickness of a four-digit NACA airfoil. At these nodes, which coincided with the solid surface, the flow was specified to be tangential to the surface. This ensured that the acoustic LEE simulation remained stable at the trailing edge, and prevented the production of spurious noise.

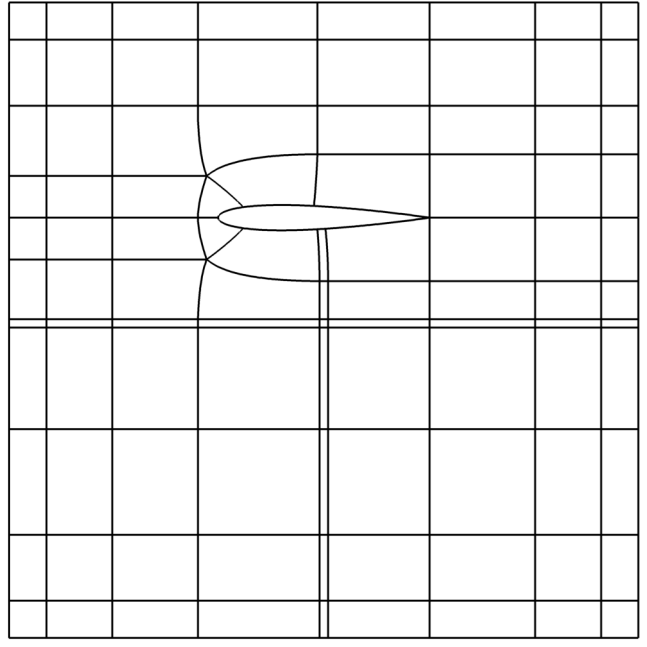

Figure 2. Topology of the NACA-0012 airfoil domain mesh for use with the LEE solver. 


\section{B. Boundary element methods}

The boundary element method is formulated in the frequency domain. The wave equation of linear acoustics can be written in terms of the time-dependent acoustic potential $\Phi_{a}(\mathbf{x}, t)$ as

$$
\frac{\partial^{2} \Phi_{a}(\mathbf{x}, t)}{\partial t^{2}}-c_{0}^{2} \nabla^{2} \Phi_{a}(\mathbf{x}, t)=0
$$

where $c_{0}$ is the speed of sound.

Under the assumption that the solution is time-harmonic such that $\Phi_{a}(\mathbf{x}, t)=\phi_{a}(\mathbf{x}) e^{-i \omega t}$, the wave equation can be reduced to the Helmholtz equation

$$
\nabla^{2} \phi_{a}(\mathbf{x})+k^{2} \phi_{a}(\mathbf{x})=0
$$

where $k=\omega / c_{0}$ is the acoustic wavenumber. The boundary condition for acoustically rigid surfaces is given by

$$
\frac{\partial \phi_{a}(\mathbf{x})}{\partial n}=\nabla \phi_{a}(\mathbf{x}) \cdot \mathbf{n}=0
$$

where $\mathbf{n}$ is the normal vector to the boundary at $\mathbf{x}$. Together, Eq. (5) and Eq. (6) may be solved to compute the sound field interior or exterior to any given boundary $S$.

The total acoustic field can be written as the sum of incident and scattered fields as $\phi_{a}(\mathbf{x})=\phi_{a}^{I}(\mathbf{x})+\phi_{a}^{S}(\mathbf{x})$. The Helmholtz equation can be reformulated to give a boundary integral equation

$$
b(\mathbf{x}) \phi_{a}(\mathbf{x})=\int_{S}\left[G(\mathbf{x}, \mathbf{y}) \frac{\partial \phi_{a}(\mathbf{y})}{\partial n}-\frac{\partial G(\mathbf{x}, \mathbf{y})}{\partial n} \phi_{a}(\mathbf{y})\right] \mathrm{d} S+\phi_{a}^{I}(\mathbf{x}),
$$

which is valid on the surface $S$, with $b(\mathbf{x})=1 / 2$ provided the boundary is smooth at $\mathbf{x}$.

For a monopole source at $\mathbf{x}_{s}$ with strength $Q$, the incident potential is given by $\phi_{a}^{I}(\mathbf{x})=Q G\left(\mathbf{x}, \mathbf{x}_{s}\right)$. This corresponds to adding a delta function $\delta\left(\mathbf{x}-\mathbf{x}_{s}\right)$ to the right hand side of Eq. (5) as a source term. The Green's function in 2-D is given by

$$
G(\mathbf{x}, \mathbf{y})=\frac{i}{4} H_{0}^{(1)}(k r)
$$

where $H_{0}^{(1)}$ is the Hankel function of the first kind of order 0 , and the radius $r=\|\mathbf{x}-\mathbf{y}\|$.

Equation (7) can then be solved using the boundary condition given by Eq. (6) to obtain the unknowns $\phi_{a}(\mathbf{y})$ on the boundary. Once these are known, the acoustic potential in the domain is computed using

$$
\phi_{a}(\mathbf{x})=\int_{S}\left[G(\mathbf{x}, \mathbf{y}) \frac{\partial \phi_{a}(\mathbf{y})}{\partial n}-\frac{\partial G(\mathbf{x}, \mathbf{y})}{\partial n} \phi_{a}(\mathbf{y})\right] \mathrm{d} S+\phi_{a}^{I}(\mathbf{x}) .
$$

The complex-valued acoustic pressure $p(\mathbf{x})$ is related to the acoustic potential $\phi_{a}(\mathbf{x})$ by

$$
p(\mathbf{x})=i \omega \rho_{0} \phi_{a}(\mathbf{x}) .
$$

Given that the solution is time-harmonic, $p_{\text {rms }}(\mathbf{x})$ is given by $|p(\mathbf{x})| / \sqrt{2}$.

The solution to the boundary integral equation given by Eq. (7) with the boundary condition given by Eq. (6), along with the computation of the acoustic pressure in the domain using Eq. (9), is conducted using the open-source solver ABEMFULL. ${ }^{a}$ ABEMFULL solves the boundary integral equations using the collocation method. The uniform and non-uniform flow formulations detailed below were implemented into the BEM solver for evaluation.

For all BEM simulations, the monopole strength was set as $Q=1$. The airfoil boundary was discretized with 120 boundary elements, which ensured at least 10 elements per wavelength and that the curvature of the leading edge was accurately represented. An acoustic BEM solution is less computationally demanding than an equivalent solution using the LEE solver. This is due to the BEM being a frequency domain method with no need to advance the solution in time, as well as unknowns needing to be computed at far fewer spatial points. Each BEM solution was obtained in less than one second on a single core of an Intel Core i7 processor at $3.4 \mathrm{GHz}$, compared to the LEE solver discussed above that computed an equivalent solution in around 15 minutes on 16 Intel Xeon cores at $2.6 \mathrm{GHz}$.

\footnotetext{
a Available from: http://www.boundary-element-method.com/
} 


\section{Uniform flow BEM approximation}

Both flow BEM approximations begin with the convected wave equation written in terms of the timedependent acoustic potential $\Phi_{a}(\mathbf{x}, t)$

$$
\left(\frac{\partial}{\partial t}+\nabla \phi_{f}(\mathbf{x}) \cdot \nabla\right)^{2} \Phi_{a}(\mathbf{x}, t)-c_{0}^{2} \nabla^{2} \Phi_{a}(\mathbf{x}, t)=0 .
$$

Here $\phi_{f}(\mathbf{x})$ is the flow potential at $\mathbf{x}$. This is solved with the boundary condition for acoustically rigid surfaces

$$
\frac{\partial \Phi_{a}(\mathbf{x}, t)}{\partial n}=0 .
$$

With the assumption that the solution is time-harmonic with $\Phi_{a}(\mathbf{x}, t)=\phi_{a}(\mathbf{x}) e^{-i \omega t}$, the convected wave equation given in Eq. (11) is reduced to the convected Helmholtz equation

$$
k^{2} \phi_{a}(\mathbf{x})+2 i \frac{k}{c_{0}} \nabla \phi_{f}(\mathbf{x}) \cdot \nabla \phi_{a}(\mathbf{x})-\frac{1}{c_{0}^{2}}\left(\nabla \phi_{f}(\mathbf{x}) \cdot \nabla\right)^{2} \phi_{a}(\mathbf{x})+\nabla^{2} \phi_{a}(\mathbf{x})=0 .
$$

Here the second and third terms are the convective terms.

With the assumption of uniform flow aligned with the $x$-axis everywhere, this can be simplified further using $\nabla \phi_{f}(\mathbf{x})=v_{0}$, and $\nabla \phi_{a}(\mathbf{x})=\partial \phi_{a}(\mathbf{x}) / \partial x$ for the convective terms. This gives

$$
k^{2} \phi_{a}(\mathbf{x})+2 i k M_{0} \frac{\partial \phi_{a}(\mathbf{x})}{\partial x}+\left[\left(1-M_{0}^{2}\right) \frac{\partial^{2} \phi_{a}(\mathbf{x})}{\partial x^{2}}+\frac{\partial^{2} \phi_{a}(\mathbf{x})}{\partial y^{2}}\right]=0,
$$

where $M_{0}=v_{0} / c_{0}$ is the Mach number. This convected Helmholtz equation can then be reduced to a regular Helmholtz equation

using the Lorentz-type transform

$$
\nabla_{\tilde{x}}^{2} \tilde{\phi}_{a}(\tilde{\mathbf{x}})+\tilde{k}^{2} \tilde{\phi}_{a}(\tilde{\mathbf{x}})=0
$$

$$
\begin{aligned}
& \mathbf{x}=(x, y) \rightarrow \tilde{\mathbf{x}}=(\tilde{x}, y), \quad \text { where } \quad \tilde{x}=\frac{x}{\sqrt{1-M_{0}^{2}}}, \\
& t \rightarrow \tilde{t}, \quad \tilde{t}=\sqrt{1-M_{0}^{2}} t+\frac{1}{\sqrt{1-M_{0}^{2}}} \frac{M_{0}}{c_{0}} x, \\
& \omega \rightarrow \tilde{\omega}, \quad \tilde{\omega}=\frac{\omega}{\sqrt{1-M_{0}^{2}}}, \\
& k \rightarrow \tilde{k}, \quad \tilde{k}=\frac{k}{\sqrt{1-M_{0}^{2}}}, \\
& \phi_{a}(\mathbf{x}) \rightarrow \tilde{\phi}_{a}(\tilde{\mathbf{x}}) e^{-i \kappa \tilde{x}}, \quad \kappa=\frac{k M_{0}}{\sqrt{1-M_{0}^{2}}}, \\
& \nabla=\left(\frac{\partial}{\partial x}, \frac{\partial}{\partial y}\right) \rightarrow \nabla_{\tilde{x}}=\left(\frac{\partial}{\partial \tilde{x}}, \frac{\partial}{\partial y}\right) .
\end{aligned}
$$

The boundary condition becomes

$$
\frac{\partial \tilde{\phi}_{a}(\tilde{\mathbf{x}})}{\partial \tilde{n}}=0
$$

The solution $\tilde{\phi}_{a}(\tilde{\mathbf{x}})$ is found in the transformed domain using the BEM technique outlined previously, where the $x$-coordinates of the boundary and source are modified using the transform given by Eqs. (16). Following the discussion by Papamoschou and Mayoral, ${ }^{4}$ the source strength is modified in the transformed domain using

$$
\tilde{Q}=\frac{Q}{i k \rho_{0} c_{0}\left(1-M_{0}^{2}\right)} .
$$

Once $\tilde{\phi}_{a}(\tilde{\mathbf{x}})$ is known, $\phi_{a}(\mathbf{x})$ may be found using $\phi_{a}(\mathbf{x})=\tilde{\phi}_{a}(\tilde{\mathbf{x}}) e^{-i \kappa \tilde{x}}$. The time-harmonic acoustic pressure is then given by

$$
p(\mathbf{x})=\rho_{0} c_{0}\left(i k-M_{0} \frac{\partial}{\partial x}\right) \phi_{a}(\mathbf{x}) .
$$

For further details of the derivation, see Papamoschou and Mayoral. ${ }^{4}$ 


\section{Non-uniform flow BEM approximation}

The non-uniform flow BEM approximation is valid under the assumptions of steady, low Mach number, irrotational, and inviscid flow. The derivation of the approximation begins, as for the uniform flow approximation, with the convected wave equation written in terms of the time-dependent acoustic potential as in Eq. (11), together with the boundary condition given by Eq. (12) for acoustically rigid surfaces.

The flow potential satisfies the Laplace equation

$$
\nabla^{2} \phi_{f}(\mathbf{x})=0
$$

and can be solved with the impermeability condition

$$
\frac{\partial \phi_{f}(\mathbf{x})}{\partial n}=0
$$

Solutions of the Laplace equation at various angles of attack and Mach numbers were obtained by implementing a solver based on panel methods, as described by Houghton and Carpenter. ${ }^{14}$ The boundary discretization used for the panel solver was the same as that for the BEM solver.

To derive the non-uniform flow BEM approximation, a source term $\delta\left(\mathbf{x}-\mathbf{x}_{s}\right)$ is added to the right hand side of Eq. (11). Using the transform given by Taylor ${ }^{5}$

$$
\begin{array}{rlrl}
\mathbf{x}=(x, y) & \rightarrow \mathbf{X}=(X, Y), & \text { where } \quad(X, Y) & =(x, y), \\
t & \rightarrow T, & T=t+\phi_{f}(\mathbf{x}) / c_{0}^{2}, \\
\Phi_{a}(\mathbf{x}, t) & \rightarrow \hat{\Phi}_{a}(\mathbf{X}, T), \\
\nabla=\left(\frac{\partial}{\partial x}, \frac{\partial}{\partial y}\right) & \rightarrow \nabla_{X}=\left(\frac{\partial}{\partial X}, \frac{\partial}{\partial Y}\right), &
\end{array}
$$

and neglecting $O\left(M_{0}^{2}\right)$ terms, the convected wave equation given in Eq. (11), with the additional source term described above, can be reduced to an ordinary wave equation

$$
\frac{\partial^{2} \hat{\Phi}_{a}(\mathbf{X}, T)}{\partial T^{2}}-c_{0}^{2} \nabla_{X}^{2} \hat{\Phi}_{a}(\mathbf{X}, T)=\delta\left(\mathbf{X}-\mathbf{x}_{s}\right) e^{i \omega \phi_{f}\left(\mathbf{x}_{s}\right) / c_{0}^{2}} e^{-i \omega T} .
$$

Under the transform, the boundary condition for acoustically rigid surfaces given in Eq. (12) becomes

$$
\frac{\partial \hat{\Phi}_{a}(\mathbf{X}, T)}{\partial n}=0
$$

Assuming that the acoustic potential is time-harmonic with $\hat{\Phi}_{a}(\mathbf{X}, T)=\hat{\phi}_{a}(\mathbf{X}) e^{-i \omega T}$, Eq. (23) can further be reduced to a Helmholtz equation

$$
\nabla_{X}^{2} \hat{\phi}_{a}(\mathbf{X})+k^{2} \hat{\phi}_{a}(\mathbf{X})=-\delta\left(\mathbf{X}-\mathbf{x}_{s}\right) e^{i \omega \phi_{f}\left(\mathbf{x}_{s}\right) / c_{0}^{2}} .
$$

Setting $\bar{\phi}_{a}(\mathbf{X})=\hat{\phi}_{a}(\mathbf{X}) e^{-i \omega \phi_{f}\left(\mathbf{x}_{s}\right) / c_{0}^{2}}$ yields a Helmholtz equation that can be solved by the BEM technique described previously

$$
\nabla_{X}^{2} \bar{\phi}_{a}(\mathbf{X})+k^{2} \bar{\phi}_{a}(\mathbf{X})=-\delta\left(\mathbf{X}-\mathbf{x}_{s}\right)
$$

with the boundary condition

$$
\frac{\partial \bar{\phi}_{a}(\mathbf{X})}{\partial n}=0
$$

The acoustic pressure is given in terms of the time-harmonic acoustic potential by

$$
p(\mathbf{x})=-\rho_{0}\left(-i \omega \bar{\phi}_{a}(\mathbf{x})+\nabla \phi_{f}(\mathbf{x}) \cdot \nabla \bar{\phi}_{a}(\mathbf{x})\right) e^{\left[i \omega\left(\phi_{f}\left(\mathbf{x}_{s}\right)-\phi_{f}(\mathbf{x})\right) / c_{0}^{2}\right]} .
$$

It can be seen that the acoustic pressure at a point $\mathbf{x}$ is only modified by $\nabla \phi_{f}(\mathbf{x})=\mathbf{u}(\mathbf{x})$ at that point, while the flow potential at the point $\mathbf{x}$ and the source point $\mathbf{x}_{s}$ modify the phase. For further details of the derivation, see Taylor ${ }^{5}$ and Agarwal and Dowling. ${ }^{6}$

The LEE can be solved to compute acoustic propagation through fully non-uniform flowfields. In contrast, the BEM uses Green's functions to compute acoustic pressure at a point. Therefore, the BEM cannot 
accurately account for propagation through a non-uniform flow. The uniform and non-uniform flow BEM formulations approximate the effects of flow on the acoustic pressure at any given observer point. For this investigation, acoustic propagation through non-uniform flows was evaluated using the LEE solver. The uniform and non-uniform flow BEM formulations were then evaluated against results from the LEE solver in order to determine which BEM flow formulation is a more accurate approximation for the observed non-uniform flow effects.

\section{Results}

\section{A. The effect of circulation on acoustic scattering}

The effect that non-uniform flow due to circulation around a lifting wing has on acoustic scattering was investigated using the LEE solver, which can account for acoustic propagation through a non-uniform flow. The effect was quantified for each angle of attack and Mach number by conducting a simulation over a uniform and a non-uniform base flow, and computing the difference between them using $\mathrm{SPL}_{\text {non-uniform }}^{\mathrm{LEE}}-\mathrm{SPL}_{\text {uniform }}^{\mathrm{LEE}}$ at each observer location.

It was described previously that the monopole source amplitude parameter $\epsilon$ was scaled so that the source strength was nominally the same between LEE and BEM simulations with a uniform flow. Figures 3 and 4 show examples of this matching of source strengths at $\alpha=5^{\circ}$, with a comparison between LEE and BEM solvers with a uniform mean flowfield at $M_{0}=0.2$ and 0.3 respectively. It can be seen that both methods are in good agreement, particularly in the $0^{\circ} \leq \theta \leq 180^{\circ}$ region, which is a region of particular interest for the scattering of landing gear noise to the ground. Similar agreement is also seen for the other angles of attack and Mach numbers, the plots of which are not presented here for brevity.

Figure 5 shows an example of an inviscid non-uniform flow solution at $M_{0}=0.2$ and $\alpha=5^{\circ}$. Figures 6 and 7 show examples of acoustic simulations at $\alpha=5^{\circ}$ over $M_{0}=0.2$ uniform and non-uniform base flows respectively. In both figures, convective amplification can be seen upstream with a compression of the acoustic wavelength, and downstream with a dilation of the wavelength. It should also be noted that these figures show the whole of the acoustic domain, and no reflections from the edge of the domain can be seen due to the use of the non-reflecting boundary conditions.

A comparison between Figures 6 and 7 shows that there is only a small effect due to the non-uniform flow at this Mach number and angle of attack. The scattering over non-uniform flow is close to that over uniform flow in the $0^{\circ} \leq \theta \leq 180^{\circ}$ region, with a slightly larger difference in the shadow region. This is most likely

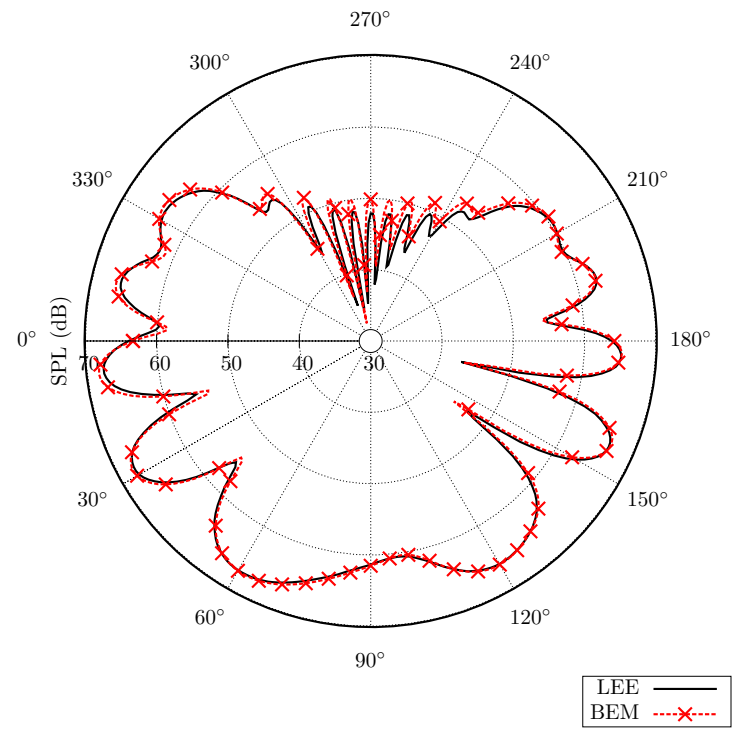

Figure 3. Comparison of SPL (dB) as computed by LEE and BEM solvers for the scattering of a monopole at $325 \mathrm{~Hz}$ by a NACA-0012 airfoil at $\alpha=5^{\circ}$ over a uniform flow with $M_{0}=0.2$.

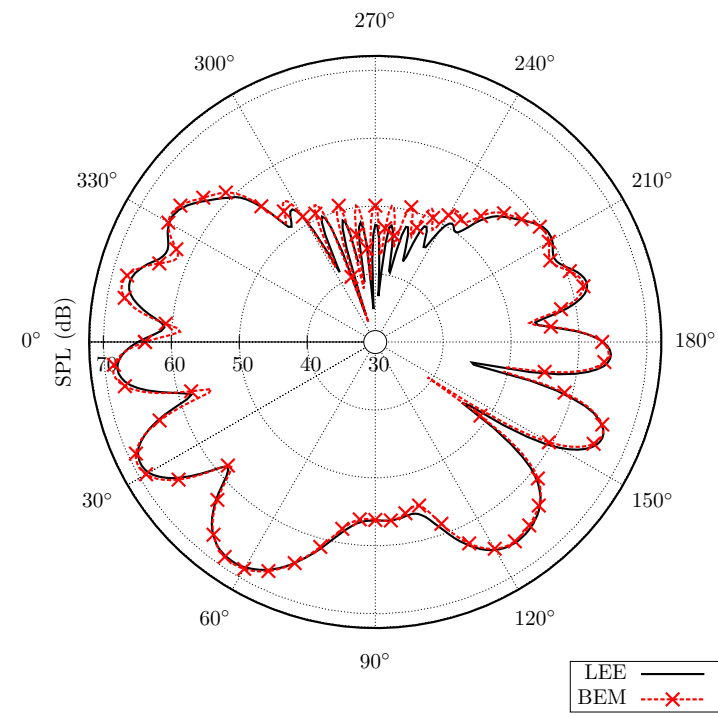

Figure 4. Comparison of SPL (dB) as computed by LEE and BEM solvers for the scattering of a monopole at $325 \mathrm{~Hz}$ by a NACA-0012 airfoil at $\alpha=5^{\circ}$ over a uniform flow with $M_{0}=0.3$. 


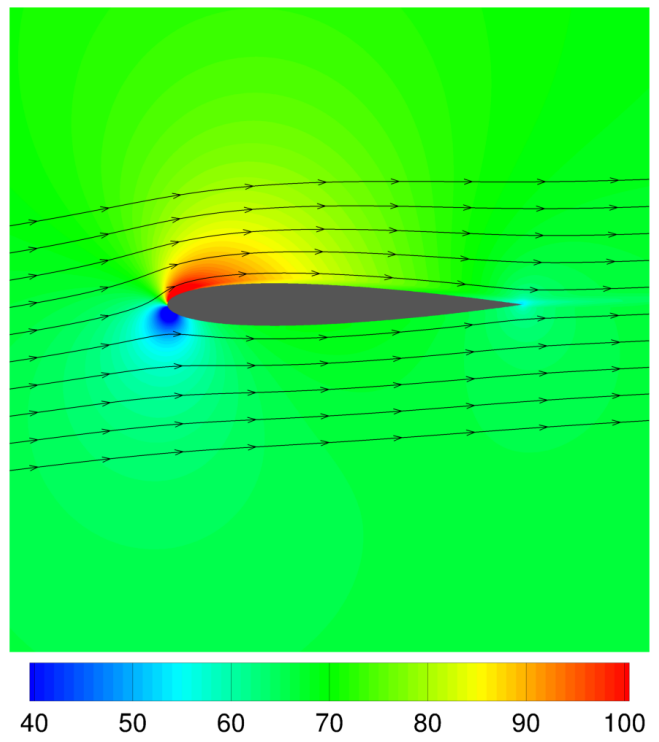

Figure 5. Flow velocity $(\mathrm{m} / \mathrm{s})$ and streamlines for a NACA-0012 airfoil at $\alpha=5^{\circ}$ in an inviscid non-uniform flow with $M_{0}=0.2$. Input flowfield for the LEE solver.

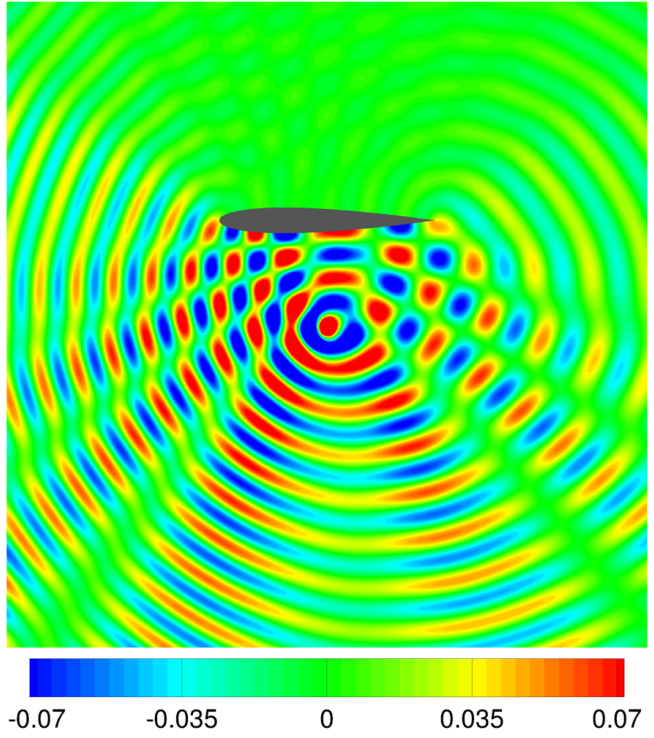

Figure 6. Acoustic pressure (Pa) for the scattering of a monopole at $325 \mathrm{~Hz}$ by a NACA-0012 airfoil at $\alpha=5^{\circ}$ over an inviscid uniform flow with $M_{0}=0.2$. Computed using the LEE solver.

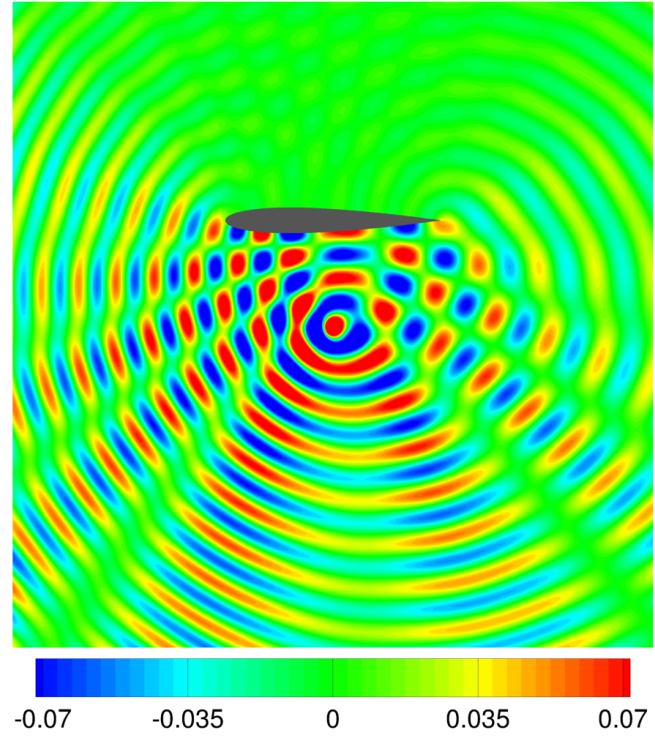

Figure 7. Acoustic pressure (Pa) for the scattering of a monopole at $325 \mathrm{~Hz}$ by a NACA-0012 airfoil at $\alpha=5^{\circ}$ over an inviscid non-uniform flow with $M_{0}=0.2$. Computed using the LEE solver.

due to diffractions around the leading and trailing edges, where there are large changes in flow velocities.

The effect of varying the angle of attack on the difference in SPL between uniform and non-uniform base flows can be seen in Figure 8. It can be seen that increasing the angle of attack from $0^{\circ}$ through to $8^{\circ}$ slightly increases the sound pressure level in the $60^{\circ} \leq \theta \leq 120^{\circ}$ region. This region is the primary region of interest for aircraft noise, as it is the region where sound is radiated towards the ground plane. The results presented in Figure 8 suggest that circulation, which increases as angle of attack increases, does have an effect on acoustic scattering, albeit relatively small. In this case, the largest increase in SPL when the angle of attack was increased from $0^{\circ}$ to $8^{\circ}$ was $1.18 \mathrm{~dB}$ at $\theta=82^{\circ}$.

This trend is further supported by Figure 9, which shows the effect of Mach number on the computed difference. Here the angle of attack was fixed and the Mach number increased, which again leads to an increase 


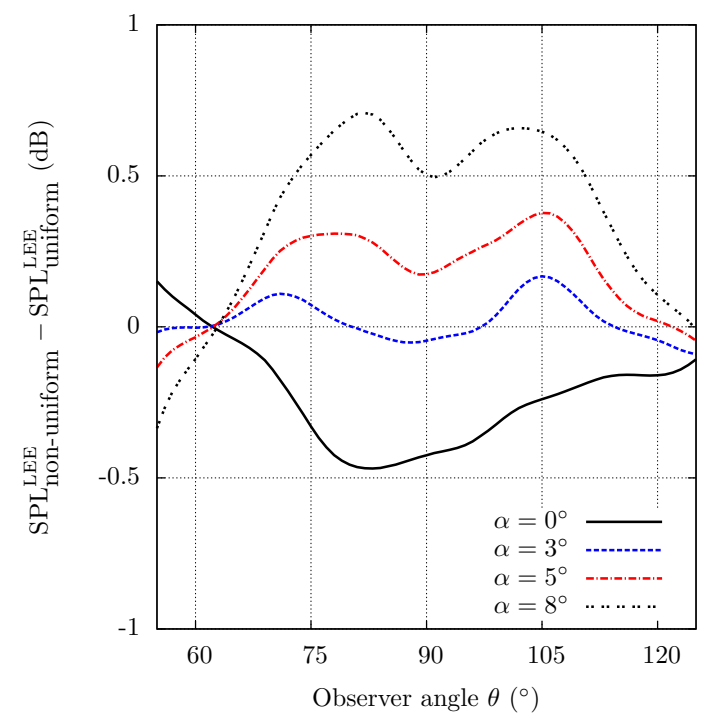

Figure 8. Difference in acoustic scattering (dB) between non-uniform and uniform base flows. Scattering of a monopole at $325 \mathrm{~Hz}$ by a NACA0012 airfoil at $M_{0}=0.2$ and varying $\alpha$.

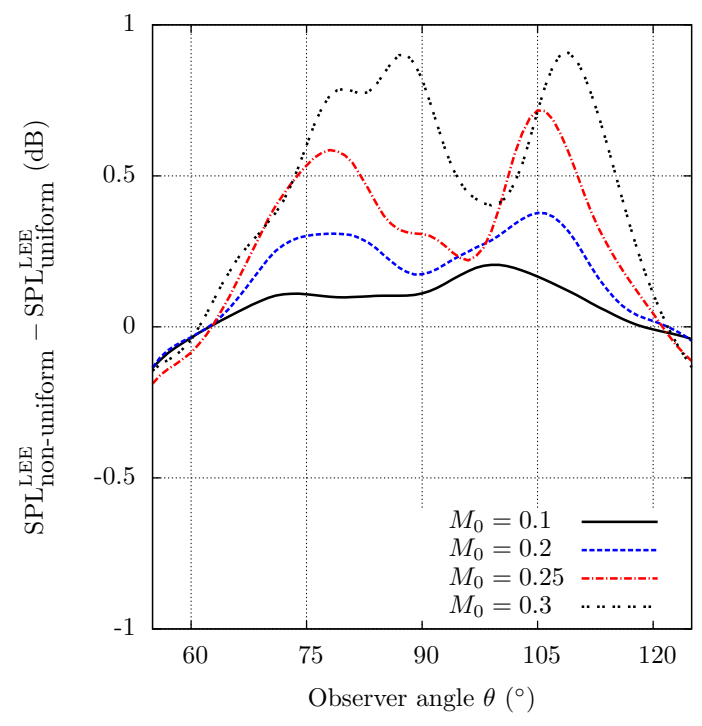

Figure 9. Difference in acoustic scattering (dB) between non-uniform and uniform base flows. Scattering of a monopole at $325 \mathrm{~Hz}$ by a NACA0012 airfoil at $\alpha=5^{\circ}$ and varying $M_{0}$.

in circulation around the wing. A similar upward trend is seen in the $60^{\circ} \leq \theta \leq 120^{\circ}$ region, although the increase in SPL at the higher Mach numbers is still less than $1 \mathrm{~dB}$. Outside of the $60^{\circ} \leq \theta \leq 120^{\circ}$ region, varying $\alpha$ or $M_{0}$ leads to small shifts in the position of directional lobes as seen in Figure 3 , which gives large differences in the regions where the lobes intersect.

The acoustic scattering of a monopole by a lifting wing has been investigated using the LEE solver, which can account for propagation through non-uniform flows. The effect of non-uniform flow caused by circulation around the lifting wing was quantified by computing the difference in SPL between uniform and non-uniform base flows at various angles of attack and Mach numbers. It was shown that increasing the angle of attack from $0^{\circ}$ to $8^{\circ}$ or Mach number from 0.1 to 0.3 , both of which increases circulation, leads to a relatively small increase in SPL of at most $1.18 \mathrm{~dB}$ in the $60^{\circ} \leq \theta \leq 120^{\circ}$ region. Results from BEM simulations using both flow formulations will now be compared to results from the LEE solver in order to evaluate the BEM flow formulations and determine which one is more accurate.

\section{B. Evaluation of boundary element method flow approximations}

To evaluate the uniform ${ }^{4}$ and non-uniform ${ }^{5,6}$ flow BEM formulations, the same simulations were conducted with the BEM solver as were done in the previous section. The uniform flow formulation uses the Lorentztype transform given in Eqs. (16) to reduce a convected wave equation as given by Eq. (11) to an ordinary Helmholtz equation, which can then be solved with regular BEM methods. It is assumed that the flow is uniform everywhere, and not modified by the scattering body. The non-uniform flow formulation uses the Taylor transform given in Eqs. (22) to also reduce Eq. (11) to an ordinary Helmholtz equation suitable for solution with regular BEM methods. For this formulation a potential flow is assumed, which can be computed through the solution of the Laplace equation given in Eq. (20).

It has already been shown in Figures 3 and 4 that having matched source strengths for the LEE and BEM simulations, the uniform flow BEM approximation is very close to that seen from the LEE solver, although slightly over-predicted in the shadow region and at some locations in the upstream direction. Figure 10 shows an example of the sound field computed with the uniform flow BEM approximation at $\alpha=5^{\circ}$ and $M_{0}=0.2$. Convective amplification effects can be observed, as they were for the previous equivalent LEE simulation shown in Figure 6.

Simulations using both flow approximations at the various Mach numbers and angles of attack were then conducted, and the results were compared to results from the LEE solver so that the formulations could be analyzed. The non-uniform flow approximation is derived by neglecting terms of $O\left(M_{0}^{2}\right)$, and the effect 
of Mach number on the accuracy of the formulation was of particular interest. An example of a potential flow solution for use with the non-uniform flow BEM formulation is shown in Figure 11. This example is at $M_{0}=0.2$ and $\alpha=5^{\circ}$.

The difference in scattering between the uniform flow BEM formulation and the non-uniform flow LEE solution at $M_{0}=0.2$ with varying $\alpha$ is shown in Figure 12. The non-uniform LEE solution fully accounts for acoustic propagation through a non-uniform flow. It can be seen that in the $60^{\circ} \leq \theta \leq 120^{\circ}$ region the difference between the solvers is less than $1 \mathrm{~dB}$.

Similarly, the difference in scattering between the non-uniform flow BEM formulation and the nonuniform flow LEE solution at $M_{0}=0.2$ with varying $\alpha$ is shown in Figure 13. Immediately it can be seen that the difference between the solvers is much greater, with the highest difference for each angle of attack being greater than $2 \mathrm{~dB}$ in the $60^{\circ} \leq \theta \leq 120^{\circ}$ region.

In both Figures 12 and 13, a downward trend in the computed difference in SPL with increasing angle of attack can be seen. This can be attributed to the slight increase in SPL seen in the non-uniform flow LEE simulations when the angle of attack is increased, as seen above in Figure 8.

Varying Mach number has a more pronounced effect on the difference between methods than varying the angle of attack. Figure 14 shows the difference in scattering between the uniform flow BEM formulation and non-uniform flow LEE solution when the Mach number is varied and the angle of attack fixed at $\alpha=5^{\circ}$. The difference between methods increases throughout the $60^{\circ} \leq \theta \leq 120^{\circ}$ region as the Mach number is increased.

Similarly, Figure 15 shows the difference in scattering between the non-uniform flow BEM formulation and non-uniform flow LEE solution when varying Mach number with $\alpha=5^{\circ}$. The non-uniform flow BEM formulation overpredicts the LEE solution in the $60^{\circ} \leq \theta \leq 120^{\circ}$ region at every Mach number. It can be seen that by $M_{0}=0.2$ the maximum difference between methods is already over $4 \mathrm{~dB}$, and by $M_{0}=0.3$ this difference is increased to over $9 \mathrm{~dB}$. The non-uniform flow BEM approximation does not capture the SPL decrease in the $60^{\circ} \leq \theta \leq 120^{\circ}$ region beneath the lifting wing at higher Mach numbers that is observed in Figure 4. This error at the higher Mach numbers under investigation can be attributed to neglecting $O\left(M_{0}^{2}\right)$ terms in the derivation of the non-uniform flow BEM formulation.

As has been been described before, the uniform flow BEM approximation is generally in good agreement with the results from the LEE solver over a uniform base flow for the Mach numbers and angles of attack investigated here. It was also shown in the preceding section that non-uniform flow due to circulation around a lifting wing only changes the observed SPL by a relatively small amount, and so the uniform flow BEM approximation is also generally in good agreement with the results from the LEE solver over a non-uniform base flow. The non-uniform flow BEM approximation was shown to be less accurate for this particular investigation than the uniform flow BEM approximation, especially when the Mach number is increased. In conclusion, the uniform flow BEM approximation is a better approximation to the effects of non-uniform flow for the particular case under investigation here.

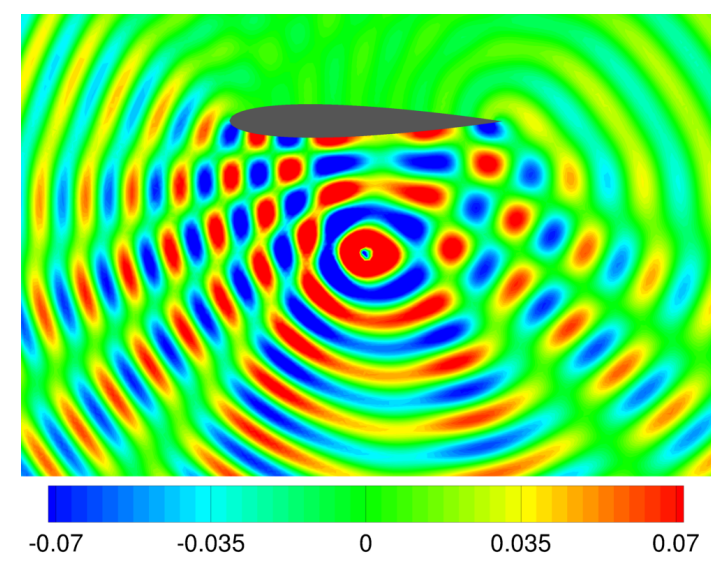

Figure 10. Acoustic pressure (Pa) for the scattering of a monopole at $325 \mathrm{~Hz}$ by a NACA-0012 airfoil at $\alpha=5^{\circ}$ over a uniform flow with $M_{0}=0.2$. Computed using the BEM solver.

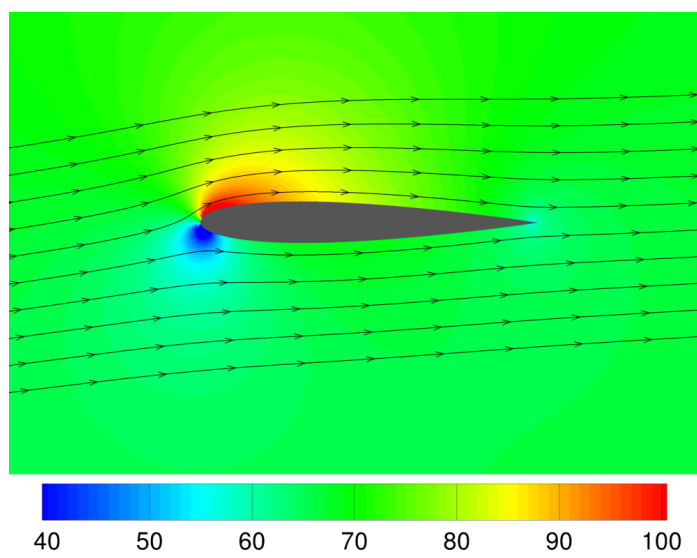

Figure 11. Flow velocity $(\mathrm{m} / \mathrm{s})$ and streamlines for a NACA-0012 airfoil at $\alpha=5^{\circ}$ in a potential non-uniform flow with $M_{0}=0.2$. Input flowfield for the BEM solver. 


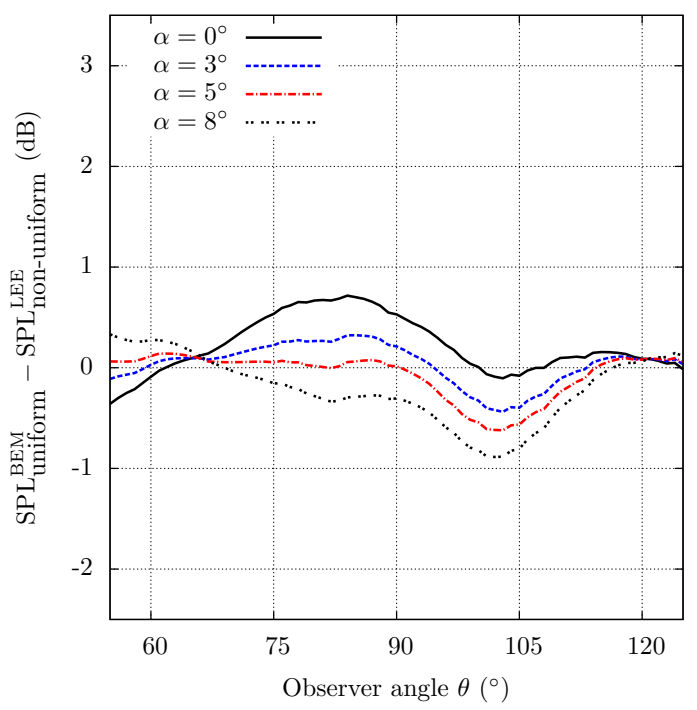

Figure 12. Difference in acoustic scattering (dB) between uniform BEM and non-uniform LEE formulations. Scattering of a monopole at $325 \mathrm{~Hz}$ by a NACA-0012 airfoil at $M_{0}=0.2$ and varying $\alpha$.

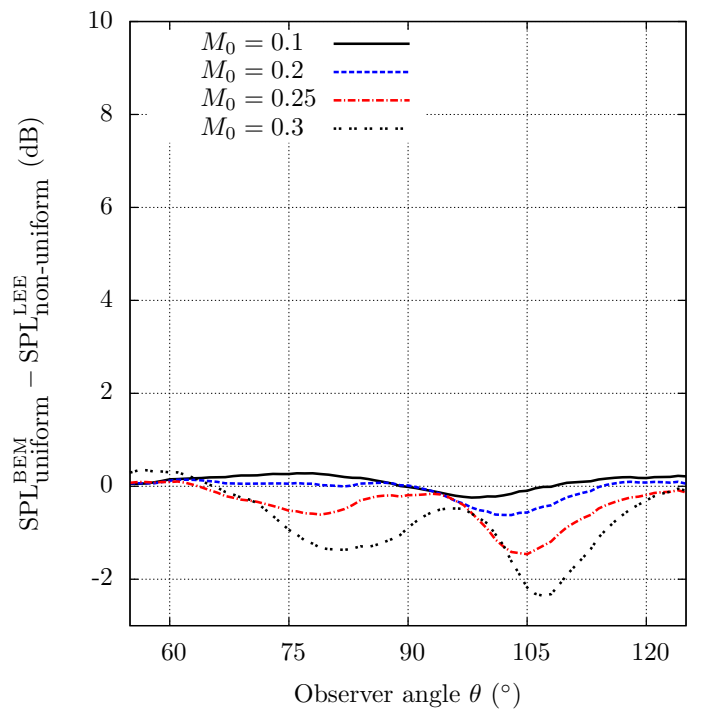

Figure 14. Difference in acoustic scattering (dB) between uniform BEM and non-uniform LEE formulations. Scattering of a monopole at $325 \mathrm{~Hz}$ by a NACA-0012 airfoil at $\alpha=5^{\circ}$ and varying $M_{0}$.

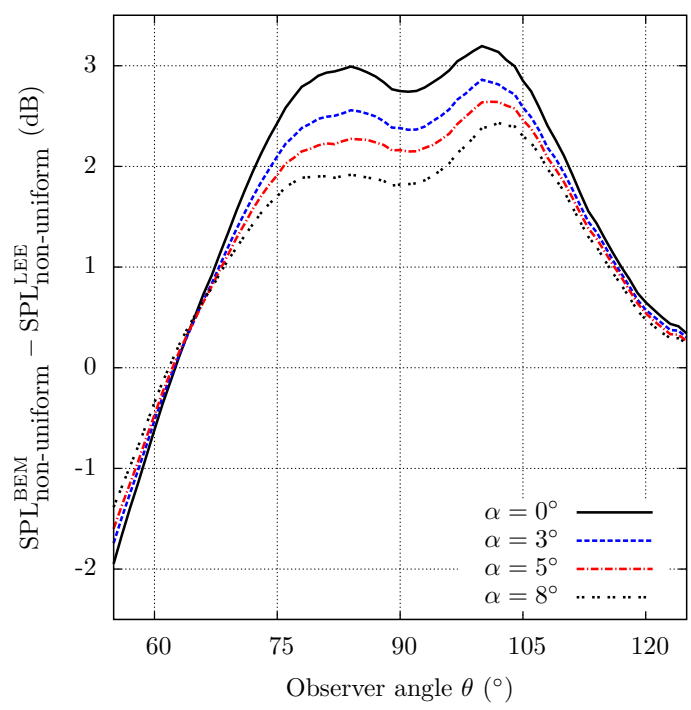

Figure 13. Difference in acoustic scattering (dB) between non-uniform BEM and non-uniform LEE formulations. Scattering of a monopole at $325 \mathrm{~Hz}$ by a NACA-0012 airfoil at $M_{0}=0.2$ and varying $\alpha$.

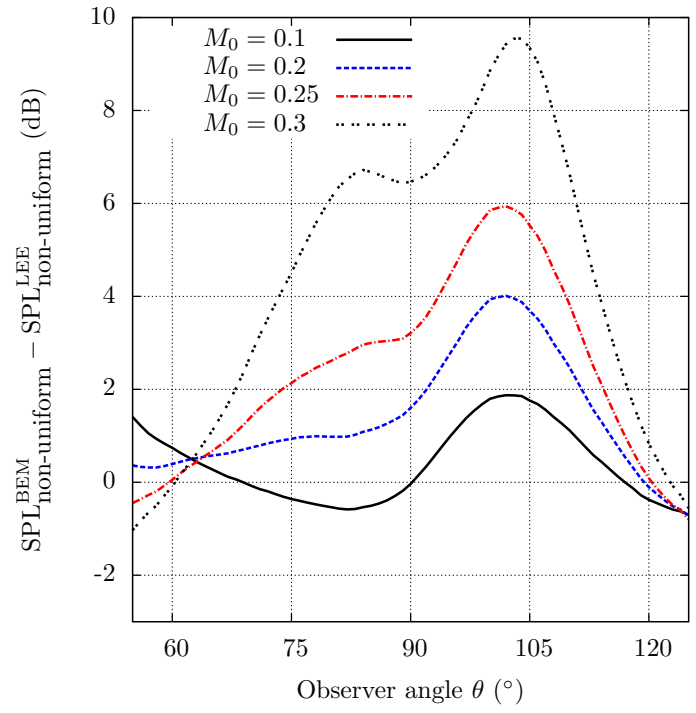

Figure 15. Difference in acoustic scattering (dB) between non-uniform BEM and non-uniform LEE formulations. Scattering of a monopole at $325 \mathrm{~Hz}$ by a NACA-0012 airfoil at $\alpha=5^{\circ}$ and varying $M_{0}$. 


\section{Conclusion}

An investigation into the scattering of an acoustic monopole by a lifting wing has been presented. The investigation was conducted using a two-dimensional test case. The monopole was located in the approximate position of a landing gear. Simulations were conducted at a single frequency using LEE and BEM solvers, with various angles of attack and Mach numbers investigated.

One of the aims of the investigation was to determine if non-uniform flow caused by circulation around a lifting wing has an effect on the scattering of landing gear noise sources. By comparing the acoustic scattering from the LEE solver over uniform and non-uniform base flows, it was seen that either increasing the angle of attack from $0^{\circ}$ to $8^{\circ}$ or increasing the Mach number from 0.1 to 0.3 , both of which increase circulation, slightly increases the SPL in the $60^{\circ} \leq \theta \leq 120^{\circ}$ region directly below the source. It is important to note that the maximum increase of SPL in this region was at most $1.18 \mathrm{~dB}$, and thus the effect is relatively small for this geometry and over the range of Mach numbers and angles of attack investigated.

The other aim of the investigation was to determine which of two flow approximations for the BEM was more suitable for the problem under consideration. It was shown that the uniform flow approximation is more accurate than the non-uniform flow approximation for this particular problem. This is due to the non-uniform flow approximation neglecting $O\left(M_{0}^{2}\right)$ terms in its derivation. Given that non-uniform flow caused by circulation around a lifting wing only has a very minor impact on the acoustic scattering, it was concluded that for this investigation the uniform flow BEM approximation could suitably predict the flow effects.

The next step in this investigation is to extend the simulations into three dimensions to ensure that any additional effects that might occur due to three-dimensional spherical propagation are accounted for. A range of frequencies will be investigated to see if the trends seen here are dependent on frequency. More realistic geometries, in particular multi-element high-lift geometries, will be investigated. The streamlines beneath the single-element airfoil investigated here only deviate slightly from the free stream. It is of interest to investigate how streamlines that are further distorted by a high-lift configuration modify the acoustic scattering of the landing gear sources.

\section{Acknowledgments}

The research funding for this project was provided by Airbus. The authors acknowledge the use of the IRIDIS High Performance Computing Facility, and associated support services at the University of Southampton, in the completion of this work.

\section{References}

\footnotetext{
${ }^{1}$ European Commission, "Flightpath 2050: Europe's Vision for Aviation," Advisory Council for Aeronautics Research in Europe, 2011.

${ }^{2}$ Dobrzynski, W., "Almost 40 Years of Airframe Noise Research: What Did We Achieve?," Journal of Aircraft, Vol. 47, No. 2, 2010, pp. 353-367.

${ }^{3}$ Zhang, X., Chen, X. X., Morfey, C. L., and Nelson, P. A., "Computation of Spinning Modal Radiation from an Unflanged Duct," AIAA Journal, Vol. 42, No. 9, 2004, pp. 1795-1801.

${ }^{4}$ Papamoschou, D., and Mayoral, S., "Modeling of Jet Noise Sources and their Diffraction with Uniform Flow," Proceedings of the 51st AIAA Aerospace Sciences Meeting, AIAA Paper 2013-0326, 2013.

${ }^{5}$ Taylor, K., "A transformation of the acoustic equation with implications for wind-tunnel and low-speed flight tests," Proceedings of the Royal Society of London. Series A, Mathematical and Physical Sciences, Vol. 363, No. 1713, 1978, pp. 271-281.

${ }^{6}$ Agarwal, A., and Dowling, A. P., "Low-Frequency Acoustic Shielding by the Silent Aircraft Airframe," AIAA Journal, Vol. 45, No. 2, 2007, pp. 358-365.

${ }^{7}$ Abbott, I. H., and von Doenhoff, A. E., "Theory of Wing Sections," Dover Publications, Inc., New York, 1959.

${ }^{8}$ Bailly, C., and Juvé, D., "Numerical Solution of Acoustic Propagation Problems Using Linearized Euler Equations," AIAA Journal, Vol. 38, No. 1, 2000, pp. 22-29.

${ }^{9}$ Kim, J. W., and Sandberg, R. D., "Efficient parallel computing with a compact finite difference scheme," Computers $\mathcal{G}$ Fluids, Vol. 58, 2012, pp. 70-87.

${ }^{10} \mathrm{Kim}$, J. W., "Optimised boundary compact finite difference schemes for computational aeroacoustics," Journal of Computational Physics, Vol. 225, 2007, pp. 995-1019.

${ }^{11} \mathrm{Kim}$, J. W., "High-order compact filters with variable cut-off wavenumber and stable boundary treatment," Computers ES Fluids, Vol. 39, 2010, pp. 1168-1182.
} 
${ }^{12} \mathrm{Hu}$, F. Q., Hussaini, M. Y., and Manthey, J. L., "Low-Dissipation and Low-Dispersion Runge-Kutta Schemes for Computational Acoustics," Journal of Computational Physics, Vol. 124, No. 1, 1996, pp. 177-191.

${ }^{13}$ Gill, J., Fattah, R., and Zhang, X., "Optimization of Non-Reflective Boundary Conditions for Aeroacoustic Simulations," Proceedings of the 21st AIAA/CEAS Aeroacoustics Conference, 2015.

${ }^{14}$ Houghton, E. L., and Carpenter, P. W., "Aerodynamics for Engineering Students," 5th ed., Elsevier ButterworthHeinemann, Oxford, England, UK, 2003. 